\title{
Distribution of injectates in the thoracic paravertebral space of the dog and cat: A cadaveric study
}

\author{
Francesco Santoro ${ }^{1 *}$, Paolo Franci ${ }^{2}$, Annamaria Grandis ${ }^{3}$ and Simond Valgimigli ${ }^{4}$ \\ ${ }^{1}$ Department of Clinical Sciences and Services, The Royal Veterinary College, Hatfield, UK \\ ${ }^{2}$ Department of Veterinary Medical Science, Largo Paolo Braccini, Grugliasco, Italy \\ ${ }^{3}$ Department of Veterinary Medical Science, University of Bologna, Ozzano dell'Emilia, Italy \\ ${ }^{4}$ Ambulatorio Veterinario S. Andrea, Lugo, Italy
}

\begin{abstract}
Background: Thoracic paravertebral block (TPVB) entails injecting a local anesthetic inside the thoracic paravertebral space (TPVS). Loss of resistance to air injection (air-LOR) was the first technique described in humans to locate the TPVS. To date, no study has investigated the spread of any substance after injection into the TPVS using the air-LOR technique nor has described the cranial and caudal limits of the space.

Aim: To identify the boundaries of the TPVS, to determine whether the air-LOR technique is reliable for the identification of the TPVS and to examine the relationship between the volume of injectate and its spread.

Methods: After a preliminary phase, the thorax of five cat and five dog cadavers was accessed and eviscerated. After TPVS probing, the polyurethane foam was injected, and the cranial and caudal borders were recorded after its maximum spread. Different volumes of a mixture of new methylene blue and ioversol were injected in the TPVS after its localization with a Tuohy needle and air-LOR technique in fourteen dog and six cat cadavers. Lateral radiographs of the vertebral column were used to document needle positioning, spread pattern and extension. The thorax of these subjects was then accessed and eviscerated to observe and record the spread of the mixture.

Results: Injecting a dye into the TPVS, localized by an air-LOR technique, resulted in multi-segmental and often bilateral subpleural staining of paravertebral, intercostal, and dorsal mediastinal structures in dog and cat cadavers. The lateral radiographs most often showed a mixed cloud-like and linear spread pattern, which could be a predictor of the longitudinal spread of the dye. The foam injected into the TPVS at the cranial and the caudal level revealed anatomical communication with the cervical, axillar, and lumbar paravertebral regions.

Conclusion: TPVS localization by air-LOR technique and injection results in a longitudinal multi-segmental spread in dog and cat cadavers. The communication of the TPVS with the axillary and lumbar regions could be of clinical interest for the brachial plexus and the lumbar intercostal nerve blocks in a clinical setting.

Keywords: Loss of resistance, Pet, Regional anesthesia, Thoracic paravertebral block, Thoracic paravertebral space.
\end{abstract}

\section{Introduction}

Several studies describe the advantages of locoregional techniques over systemic administration of analgesic drugs in terms of better analgesia and less stress response in both humans (Richman et al., 2006; Macfarlane et al., 2009) and dogs (Sibanda et al., 2006; Romano et al., 2016). Described for the first time in 1905 (Richardson and Lönnqvist, 1998), thoracic paravertebral block (TPVB) entails injecting a local anaesthetic adjacent to the thoracic vertebrae, where the spinal nerves emerge from the intervertebral foramina. Anatomically investigated and thoroughly described in humans and rats (Karmakar, 2001; Pintaric et al., 2012), but not in dogs and cats, this region is defined as the thoracic paravertebral space (TPVS), even though it can be considered only a virtual space. This area is divided into two different compartments by a thin connective layer named the endothoracic fascia, and several important nervous structures run in each of these. The subendothoracic (dorsal) compartment contains the spinal nerves and the rami communicantes, whereas in the extrapleural (ventral) one runs the sympathetic chain with its ganglia (Pintaric et al., 2012). The injection of a local anesthetic at this level results in ipsilateral somatic and sympathetic nerve blockade in multiple contiguous thoracic dermatomes cranial and caudal to the injection site (Richardson et al., 1998; Saito et al., 2001). TPVB is indicated as a perioperative anesthetic and analgesic technique in humans, mainly in breast surgery (Terkawi et al., 2015), and thoracotomy (Yeung et al., 2016). In fact, it provides equivalent analgesia with the same or fewer side effects than thoracic epidural analgesia (Davies et al., 2006; Scarci et al., 2010).

Studies on the longitudinal spread after a single injection and the cranial and caudal boundaries of TPVS in humans have produced inconsistent results (Karmakar, 2001; Bouman et al., 2017). While several 
techniques have been described for this block (Naja et al., 2011; Fujii et al., 2017; Ruscio et al., 2019), the "standard" (Richardson and Sabanathan, 1995) involved eliciting loss of resistance to air injection (air-LOR) in order to confirm the correct positioning of the Tuohy needle in the TPVS. In 2002 Saito et al. (2002) demonstrated the efficacy and lumbar spread of TPVB in rabbits, suggesting the block could be useful in Veterinary Medicine.

The literature about TPVB in small animals is increasing over time (Saito et al., 2002; Portela et al., 2012, 2017; Monticelli et al., 2017; Ferreira et al., 2018; Serra et al., 2019), yet no study to date has investigated the spread of any substance after its injection into the TPVS using the air-LOR technique or has described the cranial and caudal limits of the space. The primary aim of this study was to identify the caudo-cranial boundaries of the TPVS after injecting polyurethane foam, and to determine whether the air-LOR technique is reliable for identifying the TPVS and producing longitudinal spread in dog and cat cadavers. The secondary aim was to examine the relationship between the injected volume and the number of vertebrae stained after ioversol and new methylene blue (NMB) injection into the TPVS of dog and cat cadavers.

\section{Materials and Methods}

\section{Polyurethane foam study - preliminary phase}

Two cat and two dog cadavers of different breeds and weights were studied within 5 hours after euthanasia for clinical reasons. Animals with a history or evidence of thoracic pathologies or trauma were excluded from the study. After anatomical isolation of the region of the thoracic vertebrae (the vertebral column, muscle and skin of the dorsum, proximal tract of the ribs and dorsal mediastinum structures were left intact), the standard air-LOR technique was adopted for finding the TPVS with the aforementioned insulated body region leaning on the right lateral side. A Tuohy needle [Perican ${ }^{\circledR}$ - $B$ Braun $50 \mathrm{~mm} 18 \mathrm{G}$ for cats, $80 \mathrm{~mm} 18 \mathrm{G}$ for dogs $\leq 15$ kg body weight (BW), and $80 \mathrm{~mm} 16 \mathrm{G}$ for dogs $>15$ $\mathrm{kg} \mathrm{BW]}$ was inserted from $1.5 \mathrm{~cm}$ in the cats to $2-2.5$ $\mathrm{cm}$ in the dogs, laterally and parallel to the median plane and advanced towards the head of the rib (the first for the cranial and the thirteenth for the caudal TPVS injection). After locating the dorsal aspect of the rib's angle or the vertebral transverse process, the needle was connected with an air-filled LOR syringe, walked off the bony structure, and advanced in cranioventral direction until LOR to air injection was encountered. The polyurethane foam (diphenylmethane-4,4diisocyanate; Soudafoam ${ }^{\circledR}$, Soudal N.V., Turnhout, Belgium), prepared according to a previously described method (De Sordi et al., 2014), was slowly injected using a $20 \mathrm{ml}$ syringe until the TPVB space was fully dilated and the foam reached back to the insertion hole of the needle (approximately 10 minutes). After maximal expansion and hardening of the foam (20 minutes), the anatomical part was positioned in dorsal recumbency, and the subpleural compartments of the cranial and caudal contralateral TPVS were probed using a 9-mm 18G Tuohy needle (Perican ${ }^{\circledR}$ - B Braun) in the cats and a 2.6-mm (OD) hard disposable urinary catheter (Busters ${ }^{\circledR}$ ) in the dogs. The probes were inserted under the parietal pleura in paravertebral position and carefully advanced (avoiding pleural damage) to the first and the last costovertebral joint. To prevent significant leakage of the foam from the pleural access point during injection, the distance between the injection site and the probe tip was equal to at least half the length of the Tuohy needle in the cats and at least $15 \mathrm{~cm}$ in the dogs. The same foam of a different color was injected through the probe, and after 30 minutes the spreads obtained using the two techniques were compared. This phase was carried out in order to assess if injecting after probing or localizing with the air-LOR technique produced similar results without damaging the surrounding connective structures.

\section{Polyurethane foam study - study phase}

Five cat and five dog cadavers of various breeds and weights were studied within 5 hours after euthanasia for clinical reasons. Animals with a history or evidence of thoracic pathologies or trauma were excluded from the study. The thoracic cavity was accessed by bilateral costotomy. The heart, lungs, and thoracic tract of the trachea were removed, whereas the normal anatomy of the dorsal mediastinum was preserved. Adopting the same technique described for the preliminary phase, the subpleural compartment of the TPVS was then probed, and the polyurethane foam was slowly injected (Fig. 1) until its complete spread (approximately 10 minutes). The probes were then gently withdrawn, and the cadavers were left in position until the foam had completely expanded and hardened (20 minutes). The spread of the foam was studied by anatomical dissection. Different color stains were used to distinguish between the sides injected. The cranial and caudal borders of the TPVB were defined as the farthest vertebra reached by the colored foam for each side and direction.

\section{NMB and ioversol study}

Fourteen dog and six cat cadavers of various breeds and weights were studied within 5 hours after euthanasia for clinical reasons. The median (range) BW of the dog cadavers was $5.5 \mathrm{~kg}$ (4-9) for the seven dogs in the 5 -ml group and $24.5 \mathrm{~kg}$ (15.4-45) for the seven dogs in the 10-ml group. All dogs were dolichocephalic. The median (range) weight of the six cat cadavers was 3.55 $\mathrm{kg}(2-5)$. Animals with a history or evidence of thoracic pathologies or trauma were excluded from the study. The cadavers were positioned on the radiographic table in lateral recumbency, and the tenth thoracic spinous process was identified by palpation. After cutaneous mini cutdown, a Tuohy needle (same model and size described for the preliminary phase of the foam study) was inserted from $1.5 \mathrm{~cm}$ in the cats to $2-2.5 \mathrm{~cm}$ in the dogs laterally and parallel to the median plane and 


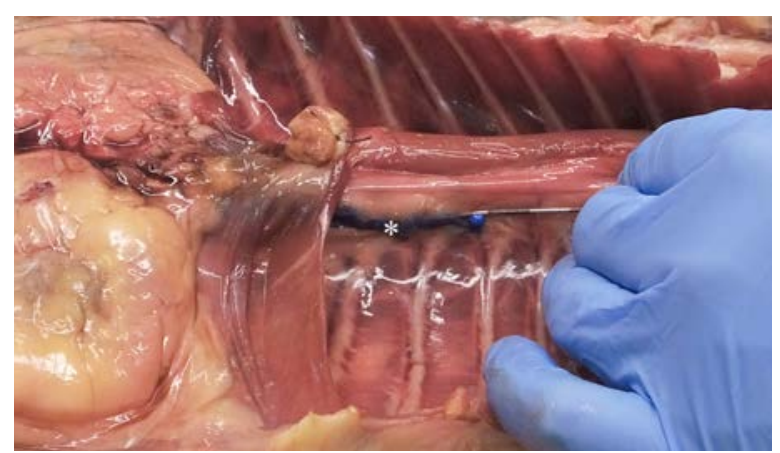

Fig. 1. Polyurethane foam injection at the level of the left caudal TPVS in a cat cadaver. The Tuohy needle has been used to probe the space, avoiding any damage to the parietal pleura (asterisk). The tip of the needle in at the level of the thirteenth left costovertebral joint.

advanced towards the tenth rib's head. After locating the dorsal aspect of the tenth rib's angle or the vertebral transverse process, the needle was connected with an air-filled LOR syringe, walked off the bony structure, and the advanced in cranioventral direction until LOR to air injection was encountered, and then a mixture (1:1) of NMB and 35\% ioversol (Optiray 350, Mallinckrodt) was injected. The total injected volume was: $3 \mathrm{ml}$ for the cats, $5 \mathrm{ml}$ for the dogs $\leq 15 \mathrm{~kg} \mathrm{BW}$, and $10 \mathrm{ml}$ for the dogs $>15 \mathrm{~kg}$ BW. The needle was left in situ after injection, and lateral radiographs of the thoracic and lumbar vertebral column were taken without moving the cadavers to document needle positioning, spread pattern, and extension of the injected mixture. Linear radiographic spread in standard lateral projection was evaluated by counting the number of vertebrae completely reached by the ioversol. A vertebra was considered to be completely reached when the contrast medium was fully extending from the cranial to caudal vertebral endplate. The cadavers were then positioned in dorsal recumbency, and the thorax was accessed by bilateral costotomy and eviscerated to observe and record the spread of the mixture under the dorsal parietal pleura and through the dorsal mediastinum. The anatomical linear spread was evaluated as the number of ipsi- and contralateral dorsal aspects of the intercostal spaces completely stained (without interruption between one costal margin and the next) in cranial and caudal directions from the space where the needle was positioned. The ninth TPVS was chosen as the site for injection for two main reasons: the intercostal spaces and spinous processes of the thoracic vertebrae are easier to be distinguished and counted at this level, and we assumed predominantly cranial spread of the dye.

\section{Statistical methods}

Data analysis was performed using GraphPad Prism Version 8.0.1 (GraphPad Prism Software, Inc., La Jolla, CA). Pearson correlation coefficients were calculated for the amount $\left(\mathrm{ml} \mathrm{kg}^{-1}\right)$ of injectate solution

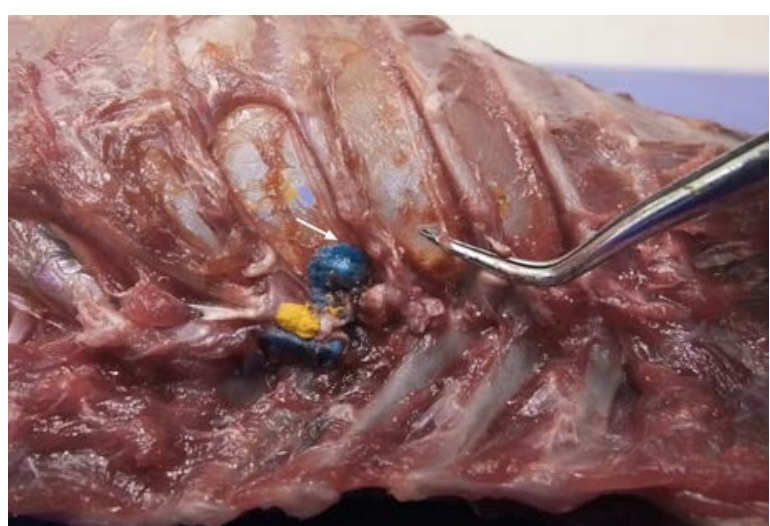

Fig. 2. Dorsal view of the thoracic cavity of a cat cadaver after a polyurethane foam injection after the removal of hepaxial and intercostal muscles. Despite the longitudinal subpleural spread, the foam surrounds the intercostal nerve only at the level of needle insertion site (arrow) and not at the adjacent spaces. The forceps are used to emphasize an intercostal nerve running above the endothoracic fascia, which appears almost completely intact in this subject.

(ioversol and NMB) and ipsilateral cranial, ipsilateral total (measured on radiography and necroscopy), and bilateral total spread, expressed as the number of stained proximal intercostal spaces. Agreement between the radiographic and the necroscopic assessment of the dye spread was analyzed using the Bland-Altman method (Bland and Altman, 1995, 2003, 2010). Results are expressed as median (range).

\section{Ethical approval}

All cadavers were donated for research purposes to the University of Bologna by the owners and, according to the Italian legislation, Ethical Approval was not necessary to perform a cadaveric study if the animals were euthanized for reasons unrelated to the study itself.

\section{Results}

\section{Polyurethane foam study}

During the preliminary phase, after post-injection anatomical dissection of the epaxial and intercostal muscles, the foam was observed to be directly in contact with the spinal nerves only at the level of the needle insertion site and not at the adjacent spaces. Cranially and caudally to the insertion point, in fact, the foam and the spinal nerves appeared separated by a thin connective layer identified as the endothoracic fascia (Fig. 2). The observed foam spread was identical between the two techniques used in this phase, and the surrounding connective tissues were intact. These findings allowed us to move to the second phase, assuming that the spread obtained after probing the TPVS could be used as a clinical model.

Cranial and caudal spread limits measured during the polyurethane foam study phase are shown in Table 1. No significant difference between left and right sides was found in both dog and cat cadavers. 
Table 1. Polyurethane foam spread limits (vertebrae).

\begin{tabular}{ccc}
\hline Dog cadavers & Cranial limit & Caudal limit \\
\hline 1 & C 3 & L 2 \\
2 & C 3 & L 3 \\
3 & C 4 & L 3 \\
4 & C 5 & L 3 \\
\hline 5 & C 4 & L 6 \\
\hline Cat cadavers & Cranial limit & Caudal limit \\
\hline 1 & C 5 & L1 \\
\hline 2 & C 5 & S 1 \\
\hline 3 & C 4 6 \\
\hline 4 & C 3 & L 3 \\
\hline 5 & C 4 & L 4 \\
\hline
\end{tabular}

Three-directional spread (cranial, lateral, and caudal) from the probe tip at the level of the first costovertebral joint was observed. At the level of the thoracic inlet, the foam had spread between the parietal pleura, the endothoracic fascia, and the longus colli muscles, in contact with the cervicothoracic ganglion and the sympathetic trunk. From this level, it then spread cranially, dorsally surrounding the left subclavian artery, and distributing within the connective tissue dorsolateral to the trachea between (and in contact with) the longus colli muscles, the trachea, the sympathetic trunk, the common carotid artery, and the esophagus. The foam was also noted to spread laterally, following the subclavian artery over the first rib, and enter the axillary space, completely surrounding the axillary artery and the visible proximal branches of the brachial plexus (Fig. 3). Caudally, the foam spread ventral to the TPVS between the parietal pleura, the endothoracic fascia, and the longus colli muscles, where it came into contact with the sympathetic trunk, the rami communicantes, and the dorsal intercostal arteries. The foam also spread ventrally, dorsal to the large blood vessels of the dorsal mediastinum. Cranial and caudal spread of the foam was observed from the probe tip at the level of the last costovertebral joint. The cranial spread at this level was identical to the aforementioned caudal spread from the cranial injection, whereas caudally it varied across subjects. The foam spread was limited to the lumbo-costal arch of the diaphragm in $3 / 5$ dogs and 4/5 cats, while in 2/5 dogs and 1/5 cats it extended past this point, distributing through the arch between the transversalis fascia and the psoas minor muscle between the quadratus lumborum and the minor psoas muscles (Figs. 4 and 5). In one dog, it spread even more caudally, between the major and the minor psoas muscles to the level of the sacral region.

\section{NMB and ioversol study}

Although with some minor intra- and inter-specific differences, a positive air-LOR test was associated with longitudinal spread along the extrapleural

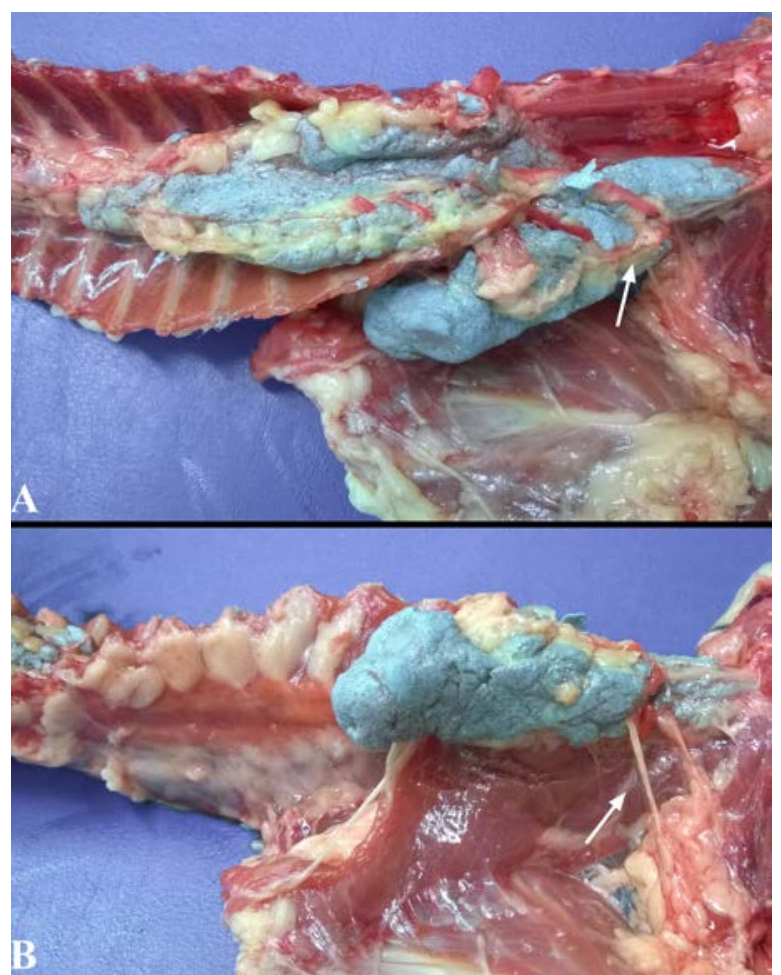

Fig. 3. Lateral spread of the polyurethane foam from the left cranial TPVS in a cat cadaver. At this level, the foam enters the axillary space, completely surrounding the axillary artery and the visible proximal branches of the brachial plexus (arrow). (A) ventral view. (B) left lateral view.

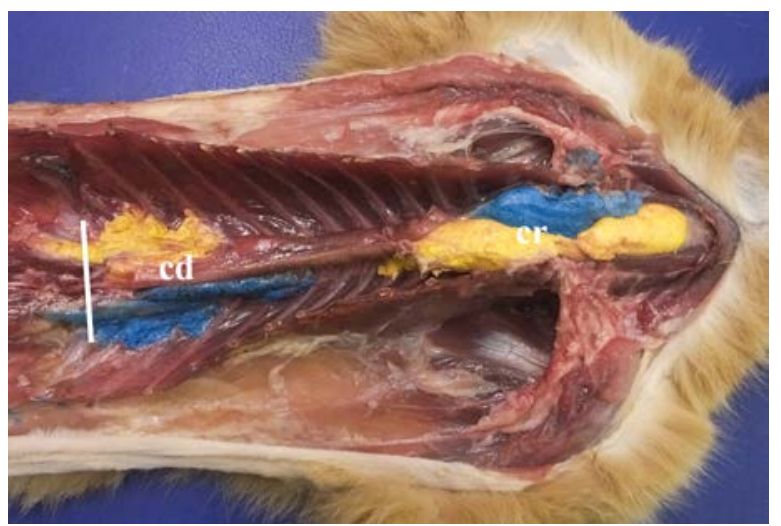

Fig. 4. Bilateral polyurethane foam spread at the level of cranial (cr) and caudal (cd) TPVS in a cat cadaver. Ventral view. The solid white line represents the level of the dorsal diaphragmatic insertion.

compartment of the TPVS in $100 \%$ of the animals. In dog cadavers, the median injected volume $\left(\mathrm{ml} \mathrm{kg}^{-1}\right)$ of the mixture was $0.6 \mathrm{ml}(0.22-1.25)$. The lateral thoracic radiographs showed multi-segmental spread in $100 \%$ of the subjects, with a median of $4.5(2-10)$ vertebrae and a distribution of $2.5(0-7)$ vertebra cranial and 1.0 (03) vertebra caudal to the injection level. A longitudinal spread pattern was noted on all radiographs, mixed 


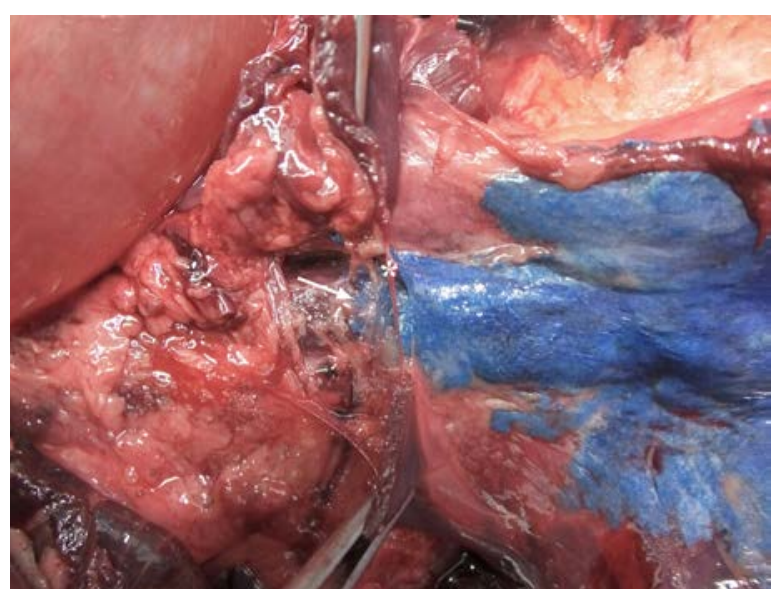

Fig. 5. Detail of polyurethane foam spread caudal to the left aspect of the dorsal diaphragmatic insertion in a dog cadaver. At this level the foam follows the anatomical communication between the endothoracic and the transversalis fascia, remaining beneath the parietal pleura and the parietal peritoneum (arrow). The diaphragm is held by the two forceps and has been almost completely removed at the level of the foam passage (centre of the image), where only his thin insertion is still visible (asterisk).

with a cloudy pattern in 11/14 (78\%) images and with an intercostal pattern at the level of the ninth intercostal space in 1/14 (7\%) image (Fig. 6). Necropsy revealed multi-segmental ipsilateral longitudinal spread in 100\% of the subjects (Fig. 7). The median stained intercostal spaces were $6.5(4-11)$, with 5 or more spaces stained in $78 \%$, whereas contralateral longitudinal spread was detected in 9/14 (64.3\%) spaces, with a median of 2.5 (0-6) (Fig. 8). The median cranial versus caudal spread ipsilateral and contralateral to the injection site was 3 (1-7) versus $2(0-3)$ and $0(0-4)$ versus $1(0-2)$ space, respectively. Distributions of $\mathrm{NMB}$ and ioversol after each mixture injection are shown in Figure 9. The only statistically significant correlation was between the radiographical and the necroscopic assessment of the longitudinal spread in the dog cadavers $(p=0.0028)$. In cat cadavers, the median injected volume ( $\mathrm{ml} \mathrm{kg}^{-1}$ ) was $0.84 \mathrm{ml}(0.6-1.5)$. The lateral thoracic radiographs disclosed multi-segmental spread in all of the subjects, with a median of 5 (2-6) vertebrae and distribution of 2 (0-3) vertebrae cranial and 2 (1-2) vertebrae caudal to the injection level. A mix of cloudy and linear spread patterns was noted on all radiographs, with the concurrent presence of an intercostal pattern at the level of the ninth intercostal space in 1/6 (17\%) image (Fig. 10). Necropsy revealed multi-segmental ipsilateral longitudinal spread in $100 \%$ of the subjects. The median number of stained intercostal spaces (dorsal aspect) was 6.5 (5-7), with 5 or more spaces stained in all subjects, whereas contralateral longitudinal spread was seen in $5 / 6(83.3 \%)$ subjects, with a median of 5 (0-7) (Fig. 11). The median distribution of the cranial vs. caudal

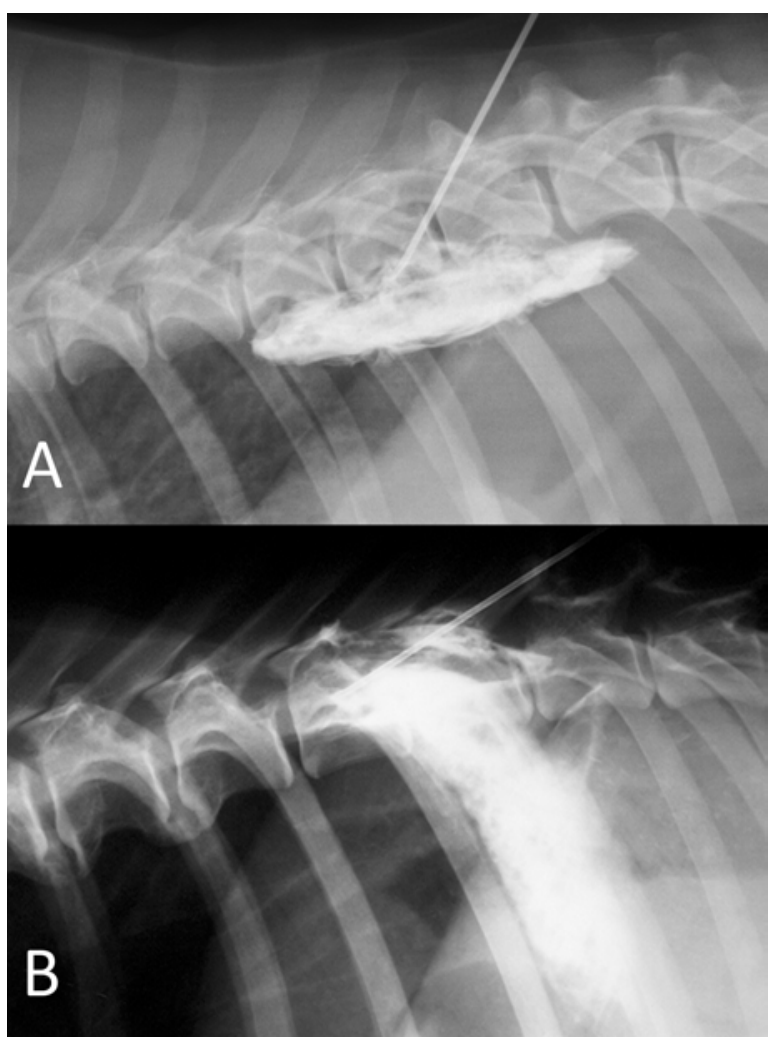

Fig. 6. NMB and ioversol mixed longitudinal and cloud-like (A) and intercostal (B) spread pattern in a lateral radiograph of a dog cadaver.

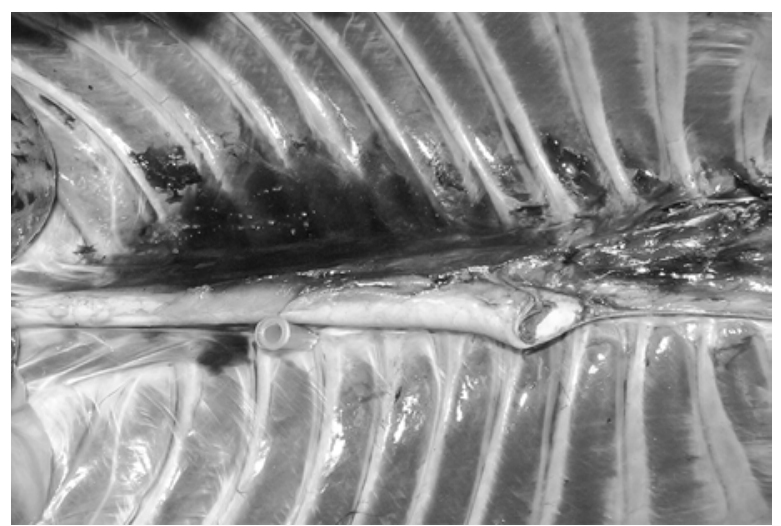

Fig. 7. NMB and ioversol longitudinal ipsilateral thoracic paravertebral spread in a dog cadaver. Ventral view.

spread ipsilateral and contralateral to the injection site was 3 (2-4) versus $2.5(1-3)$ spaces and $2(0-4)$ versus $2(0-3)$ spaces, respectively. Staining of the parietal pleura surrounding the dorsal mediastinum, the so called pre-vertebral space and the proximal intercostal spaces were observed in all subjects, regardless of the volume or the spread pattern. Distributions of NMB and ioversol after each mixture injection are shown in Figure 12. 


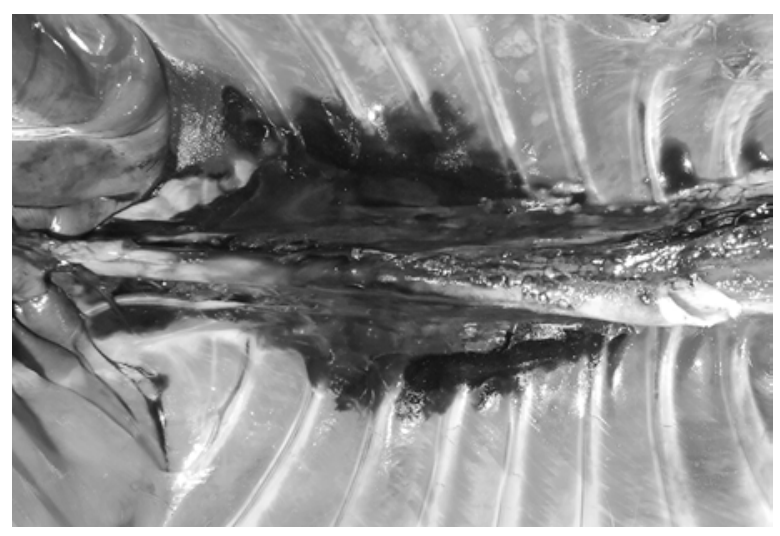

Fig. 8. NMB and ioversol longitudinal bilateral thoracic paravertebral spread in a dog cadaver. Ventral view.

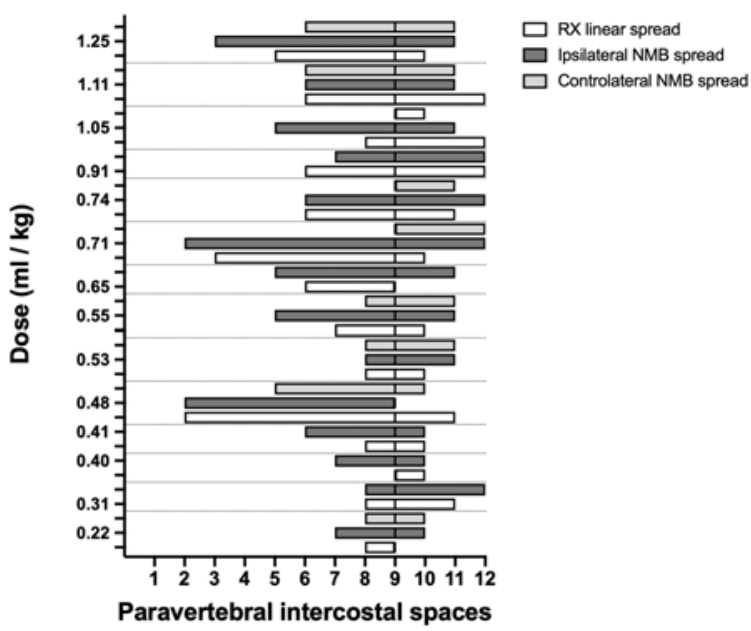

Fig. 9. Staining and contrast distribution following increasing doses of 1:1 mixture of NMB and ioversol injected in single shot at the level of the ninth intercostal space in dog cadavers. The solid line at the level of the ninth paravertebral intercostal space represents the injection site.

\section{Discussion}

To our knowledge, this is the first study investigating the cranial and caudal limits of TPVB in dog and cat cadavers. For this purpose, we used polyurethane foam because of its advantages over liquid dyes and resins: by virtue of its chemical and physical properties, the foam spreads easily within anatomical cavities and naturally communicating anatomical spaces, without damaging anatomical structures or staining surrounding tissues (De Sordi et al., 2014). The foam spread to the brachial plexus and the retroperitoneal space, demonstrating ways of anatomical communication that are of interest for investigating new approaches to brachial plexus and thoraco-lumbar paravertebral blocks in dogs and cats.

The caudal limit of the TPVS has been a debated topic because of inconsistent study results. Some studies identified the origin of the psoas major muscle as the caudal boundary of the TPVS (Lönnqvist and Hildingsson, 1992), whereas others claimed

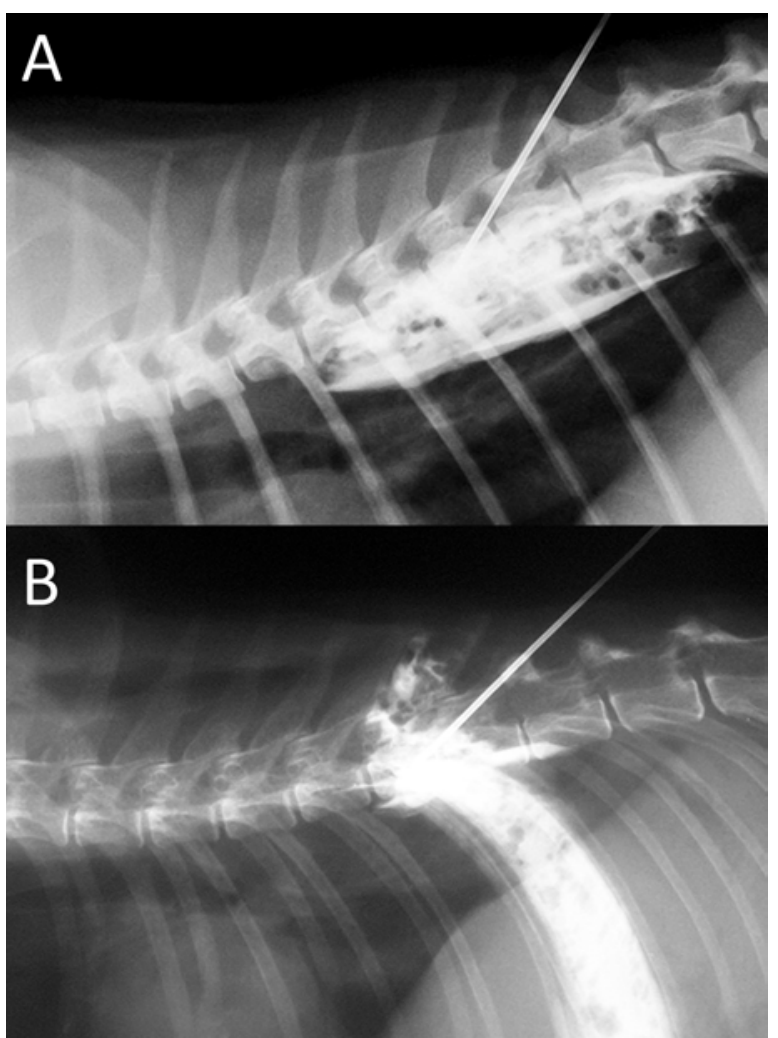

Fig. 10. NMB and ioversol mixed longitudinal and cloud-like (A) and intercostal (B) spread pattern in a lateral radiograph of a cat cadaver.

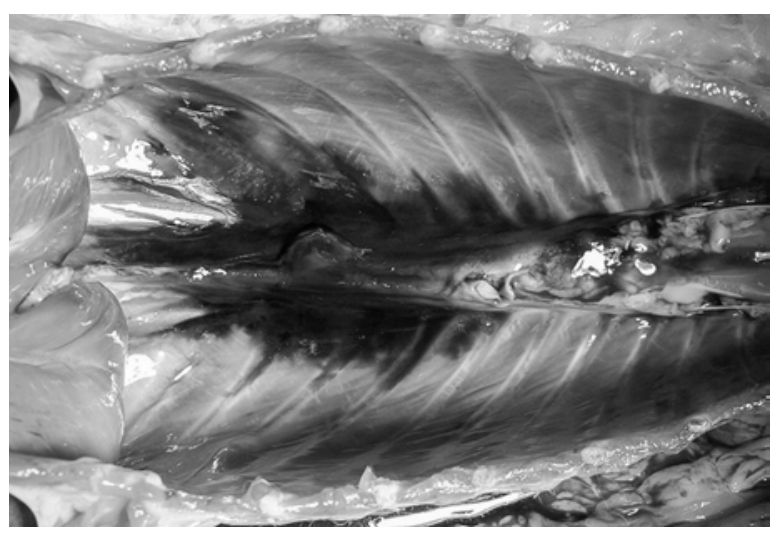

Fig. 11. NMB and ioversol longitudinal bilateral thoracic paravertebral spread in a cat cadaver. Ventral view.

observation of lumbar spread of medium injected in the caudal portion of the space in humans, rabbits, and dogs (Conacher and Kokri, 1987; Saito et al., 2002; Serra et al., 2019), which is probably explained by anatomical communication between the endothoracic and the transversalis fascia, the basis of the "extended unilateral anesthesia" (Saito et al., 1999). This anatomical feature underlies the rationale for the use of TPVB in inferior abdominal surgery in humans (Fusco et al., 2016). Interestingly, our findings disclosed a 


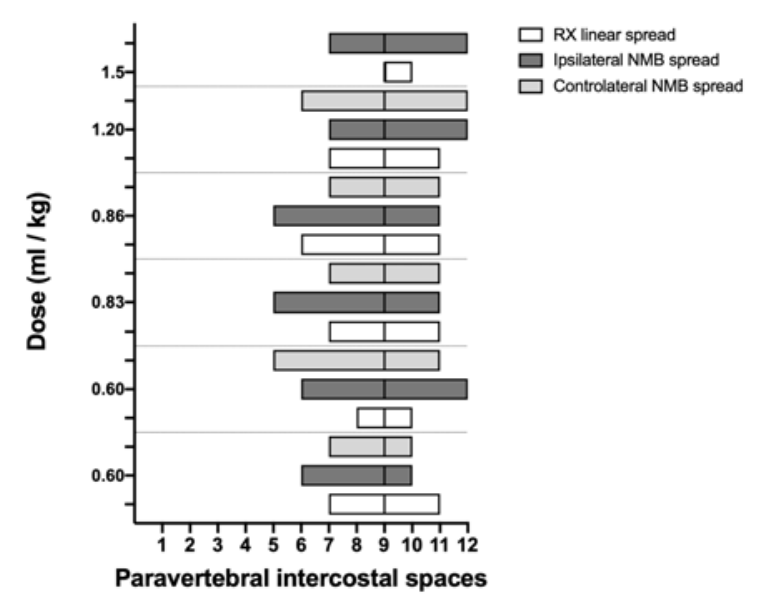

Fig. 12. Staining and contrast distribution following increasing doses of 1:1 mixture of NMB and ioversol injected in single shot at the level of the ninth intercostal space in cat cadavers. The solid line at the level of the ninth paravertebral intercostal space represents the injection site.

similar communication between the thoracic and the caudal lumbar PVS in the dog and the cat cadavers, although abdominal retroperitoneal spread was not consistent across all subjects. In our opinion, reaching the lumbar paravertebral spaces with a single caudal thoracic injection could be useful in invasive unilateral surgeries such as radical mastectomy or oncological surgery of the caudal thoracic wall or the flank region. The cranial spread limit observed at the level of the third - fifth cervical vertebra in the dog and the cat cadavers is in line with the human literature (Karmakar, 2001), although the cranial boundary of the TPVS in humans is still poorly described. The present study confirms what is already known (Portela et al., 2012, 2017; Serra et al., 2019) about the anatomical contiguity between the TPVS and the prevertebral, mediastinal, and intercostal space. Not having investigated the staining of the epidural space can be considered one of the major limitations of this study.

Despite its expansivity, polyurethane foam has been proven to exert limited pressure on the surrounding tissues. In fact, when injected inside renal and pulmonary vasculature and inside the airways, after its hardening, anatomical structures such as pulmonary alveolar units and small venules were left intact and still fully recognizable (De Sordi et al., 2014). This considered, although unlikely, it is impossible to completely exclude that the physical properties of the foam could have influenced the anatomical results of this study. Based on the results of the preliminary phase and a study of the TPVS in human cadavers (Lönnqvist and Hildingsson, 1992), we performed transpleural probing of the TPVS rather than using the clinical approach in order to avoid inadvertent piercing of the dorsal parietal pleura during high-pressure injection. This non-clinical approach may be considered another limitation of our study.
Although clinical studies of the TPVB in dogs and cats are lacking, multi-segmental longitudinal spread after a single injection of a local anesthetic could have several theoretical advantages over a multiple injection technique: reduction of injection-associated complications and shorter execution time.

In the present study, the use of the air-LOR technique to inject a fluid into the TPVS consistently resulted in the multi-segmental longitudinal spread, which was five vertebrae lengths in almost $80 \%$ of the subjects, with major distribution cranial to the injection site. The longitudinal spread confirms previous findings in human and animal studies that used LOR, ultrasound, or electrical nerve stimulation (ENS) (Conacher and Kokri, 1987; Naja et al., 2004; Cowie et al., 2010; Portela et al., 2012; Monticelli et al., 2017; Serra et al., 2019). Despite the longitudinal multi-segmental spread, we found no direct contact between the foam and the intercostal nerves cranial or caudal to the injection site. This finding may support the influence of the endothoracic fascia on the spread of the injectate, as previously reported (Portela et al., 2017). We found no statistically significant correlation between the volume of the dye and its linear spread. Although a similar finding has been described in human studies (Conacher and Kokri, 1987; Cheema et al., 2003; Kotzé et al., 2009), the small sample size of our study could have influenced this result. In 4/14 dogs (28.6\%) and $3 / 6(50 \%)$ cats the dye reached and crossed the twelfth intercostal space in some cases. Therefore, it is likely that we somehow underestimated the extent of the potential for the caudal spread of the dye. Our observation of three main radiographic spread patterns (cloud-like, intercostal, and longitudinal), often combined, is shared by a previous study in humans (Naja et al., 2004). The anatomical complexity of the TPVS seems to be the key to understand the high variability of patterns after a single injection of local anesthetic. It has been hypothesized that the space-locating technique could be correlated with a specific pattern (Naja et al., 2004). In humans, the ENS-guided technique is not reliable in producing longitudinal spread and exposes the patient to risks and prolonged execution time due to multiple injections (Naja et al., 2004; Naja and Lönnqvist, 2008). Similarly, in a study in dog cadavers, the authors observed no longitudinal spread using the ENS-guided technique (Portela et al., 2012). When using a blind loco-regional technique, it could be useful to have a predictor of correct needle positioning and block extension, as well. To our knowledge, no study involving humans or animals has compared dye spread by radiographic and by post-dissection examination. We found a statistical correlation between the results of the two methods only for the longitudinal spread in the dogs; however, our defining criteria for the spread extension and the presence of partially stained intercostal spaces and vertebral bodies may have led to its underestimation in some subjects. The post-dissection subpleural linear dye spread in one 
cadaver where only radiographic intercostal spread was observed, suggests that x-ray pattern could be a poor predictor of the actual longitudinal distribution of local anesthetic in some patients. For this study, x-rays were taken immediately after injection of ioversol and NMB in the TPVB. Despite the lack of supporting data, it is reasonable to hypothesize that the timing between injection and image acquisition may have influenced the results. Because of the cadaveric model, the different viscosity between local anesthetics and the substances used in this study, and because of the in vivo difference between post-injection imaging and sensory block spread (Marhofer et al., 2013), no conclusion about its potential clinical use can be made.

\section{Conclusion}

The air-LOR technique was proven effective for the localization of the extrapleural compartment of the TPVS, and the injection of a dye at this level resulted in multi-segmental and often bilateral subpleural staining of paravertebral, intercostal, and dorsal mediastinal structures in dog and cat cadavers. The lateral radiographic images most often showed a mixed cloud-like and linear spread pattern, which could be a predictor of the extension of longitudinal spread of the dye. The foam injection into the TPVS at the cranial and the caudal level revealed anatomical communication with the cervical, axillar, and lumbar paravertebral regions. Notwithstanding the cadaveric nature of the study, these findings may be of potential clinical interest. Further anatomical and clinical studies are needed to better understand the factors involved in this unique and potentially useful nerve block in small animal practice.

\section{Conflict of interest}

The authors declare that they have no conflict of interest.

\section{Authors' contributions}

All the authors contributed to the study conception and design. Material preparation and data collection were performed by Francesco Santoro, Annamaria Grandis and Simond Valgimigli. Data analysis was performed by Francesco Santoro and Paolo Franci. The manuscript was written by Francesco Santoro and approved by all the authors.

\section{References}

Bland, J.M. and Altman, D.G. 1995. Comparing methods of measurement: why plotting difference against standard method is misleading. Lancet. 346, 1085-1087.

Bland, J.M. and Altman, D.G. 2003. Applying the right statistics: Analyses of measurement studies. Ultrasound Obstet. Gynecol. 22, 85-93.

Bland, J.M. and Altman, D.G. 2010. Statistical methods for assessing agreement between two methods of clinical measurement. Int. J. Nurs. Stud. 47, 931936.
Bouman, E.A.C., Sieben, J.M., Balthasar, A.J.R., Joosten, E.A., Gramke, H.F., van Kleef, M. and Lataster, A. 2017. Boundaries of the thoracic paravertebral space: potential risks and benefits of the thoracic paravertebral block from an anatomical perspective. Surg. Radiol. Anat. 39, 1117-1125.

Cheema, S., Richardson, J. and McGurgan, P. 2003. Factors affecting the spread of bupivacaine in the adult thoracic paravertebral space. Anaesthesia. 58, 684-687.

Conacher, I.D. and Kokri, M. 1987. Postoperative para vertebral blocks for thoracic surgery: a radiological appraisal. Br. J. Anaesth. 59, 155-161.

Cowie, B., McGlade, D., Ivanusic, J. and Barrington, M.J. 2010. Ultrasound-guided thoracic paravertebral blockade: a cadaveric study. Anaesth. Analg. 110, 1735-1739.

Davies, R.G., Myles, P.S. and Graham, J.M. 2006. A comparison of the analgesic efficacy and sideeffects of paravertebral vs epidural blockade for thoracotomy - A systematic review and metaanalysis of randomized trials. Br. J. Anaesth. 96, 418-426.

De Sordi, N., Bombardi, C., Chiocchetti, R., Clavenzani, P., Trerè, C., Canova, M. and Grandis, A. 2014. A new method of producing casts for anatomical studies. Anat. Sci. Int. 89, 255-265.

Ferreira, T.H., Teixeira, L.B.C., Schroeder, C.A., de Miguel Garcia, C. and Schroeder, K.M. 2018. Description of an ultrasound-guided thoracic paravertebral block technique and the spread of dye in dog cadavers, Vet. Anaesth. Analg. 45, 811-819.

Fujii, T., Shibata, Y., Ban, Y., Shitaokoshi, A. and Nishiwaki, K. 2017. Catheterization in an ultrasound-guided thoracic paravertebral block using thoracoscopy. Asian. J. Anesthesiol. 55, 24-25.

Fusco, P., Cofini, V., Petrucci, E., Scimia, P., Paladini, G., Behr, A.U., Gobbi, F., Pozone, T., Danelli, G., Di Marco, M., Vicentini, R., Necozione, S. and Marinangeli, F. 2016. Unilateral paravertebral block compared with subarachnoid anesthesia for the management of postoperative pain syndrome after inguinal herniorrhaphy: a randomized controlled clinical trial. Pain 157, 1105-1113.

Karmakar, M.K. 2001. Thoracic paravertebral block. Anesthesiology. 95, 771-780.

Kotzé, A., Scally, A. and Howell, S. 2009. Efficacy and safety of different techniques of paravertebral block for analgesia after thoracotomy: a systematic review and metaregression. Br. J. Anaesth. 103, 626-636.

Lönnqvist, P.A. and Hildingsson, U. 1992. The caudal boundary of the thoracic paravertebral space: a study in human cadavers. Anaesthesia. 47, 1051-1052.

Macfarlane, A.J.R., Prasad, G.A., Chan, V.W.S. and Brull, R. 2009. Does regional anaesthesia improve outcome after total hip arthroplasty? A systematic review. Br. J. Anaesth. 103, 335-345. 
Marhofer, D., Marhofer, P., Kettner, S.C., Fleischmann, E., Prayer, D., Schernthaner, M., Lackner, E., Willschke, H., Schwetz, P. and Zeitlinger, M. 2013. Magnetic resonance imaging analysis of the spread of local anesthetic solution after ultrasound-guided lateral thoracic paravertebral blockade: a volunteer study. Anesthesiology. 118, 1106-1112.

Monticelli, P., Jones, I. and Viscasillas, J. 2017. Ultrasound-guided thoracic paravertebral block: cadaveric study in foxes (Vulpes vulpes). Vet. Anaesth. Analg. 44, 968-972.

Naja, Z.M. and Lönnqvist, P.A. 2008. Somatic paravertebral nerve blockade. Incidence of failed block and complications. Anaesthesia. 56, 11841188.

Naja, Z.M., Naccache, N., Ziade, F., El-Rajab, M., Itani, T. and Baraka, A. 2011. Multilevel nerve stimulator-guided paravertebral block as a sole anesthetic technique for breast cancer surgery in morbidly obese patients. J. Anesth. 25, 760-764.

Naja, Z.M., Ziade, F.M., El-Rajab, M., El Tayara, K. and Lönnqvist, P.A. 2004. Varying anatomical injection points within the thoracic paravertebral space: effect on spread of solution and nerve blockade. Anaesthesia. 59, 459-463.

Pintaric, T.S., Veranic, P., Hadzic, A., Karmakar, M. and Cvetko, E. 2012. Electron-microscopic imaging of endothoracic fascia in the thoracic paravertebral space in rats. Reg. Anesth. Pain Med. 37, 215-218.

Portela, D.A., Campoy, L., Otero, P.E., Martin-Flores, M. and Gleed, R.D. 2017. Ultrasound-guided thoracic paravertebral injection in dogs: a cadaveric study. Vet. Anaesth. Analg. 44, 636-645.

Portela, D.A., Otero, P.E., Sclocco, M., Romano, M., Briganti, A. and Breghi, G. 2012. Anatomical and radiological study of the thoracic paravertebral space in dogs: iohexol distribution pattern and use of the nerve stimulator. Vet. Anaesth. Analg. 39, 398-408.

Richardson, J. and Lönnqvist, P.A. 1998. Thoracic paravertebral block. Br. J. Anaesth. 81, 230-238.

Richardson, J. and Sabanathan, S. 1995. Thoracic paravertebral analgesia. Acta Anaesthesiol. Scand. 39, 1005-1015.

Richardson, J., Jones, J. and Atkinson, R. 1998. The effect of thoracic paravertebral blockade on intercostal somatosensory evoked potentials. Anesth. Analg. 87, 373-376.

Richman, J.M., Liu, S.S., Courpas, G., Wong, R., Rowlingson, A.J., McGready, J., Cohen, S.R. and Wu, C.L. 2006. Does continuous peripheral nerve block provide superior pain control to opioids? A meta-analysis. Anesth. Analg. 102, 248-257.
Romano, M., Portela, D.A., Breghi, G. and Otero, P.E. 2016. Stress-related biomarkers in dogs administered regional anaesthesia or fentanyl for analgesia during stifle surgery. Vet. Anaesth. Analg. 43, 44-54.

Ruscio, L., Renard, R., Lebacle, C., Zetlaoui, P., Benhamou, D. and Bessede, T. 2019. Thoracic paravertebral block: comparison of different approaches and techniques. A study on 27 human cadavers. Anaesth. Crit. Care Pain Med. 39(1), 53-58; doi:10.1016/j.accpm.2019.04.003Saito, T., Den, S., Cheema, S.P.S., Tanuma, K., Carney, E., Carlsson, C. and Richardson, J. 2001. A singleinjection, multi-segmental paravertebral blockextension of somatosensory and sympathetic block in volunteers. Acta Anaesthesiol. Scand. 45, 30-33.

Saito, T., Den, S., Tanuma, K., Tanuma, Y., Carney, E. and Carlsson, C. 1999. Anatomical bases for paravertebral anesthetic block: fluid communication between the thoracic and lumbar paravertebral regions. Surg. Radiol. Anat. 21, 359-363.

Saito, T., Tanuma, K., Tanuma, Y., Futami, C., Shichinohe, K., Shimizu, M., Sasako, M., Shafland, J., Wang, B.C., Carlsson, C. and Carney, E. 2002. Appendectomy in rabbits with extended unilateral anesthesia. Exp. Anim. 51, 63-68.

Scarci, M., Joshi, A. and Attia, R. 2010. In patients undergoing thoracic surgery is paravertebral block as effective as epidural analgesia for pain management? Interact. Cardiovasc. Thorac. Surg. 10, 92-96.

Serra, R.M., Jimenez, C.P., Monticelli, P., Plested, M. and Viscasillas, J. 2019. Assessment of an ultrasound-guided technique for catheterization of the caudal thoracic paravertebral space in dog cadavers. Open Vet. J. 9, 230-237.

Sibanda, S., Hughes, J.M.L., Pawson, P.E., Kelly, G. and Bellenger, C.R. 2006. The effects of preoperative extradural bupivacaine and morphine on the stress response in dogs undergoing femoro-tibial joint surgery. Vet. Anaesth. Analg. 33, 246-257.

Terkawi, A.S., Tsang, S., Sessler, D.I., Terkawi, R.S., Nunemaker, M.S., Durieux, M.E. and Shilling, A. 2015. Improving analgesic efficacy and safety of thoracic paravertebral block for breast surgery: a mixed-effects meta-analysis. Pain Physician. 18, 757-780.

Yeung, J.H., Gates, S., Naidu, B.V., Wilson, M.J. and Gao Smith, F. 2016. Paravertebral block versus thoracic epidural for patients undergoing thoracotomy. Cochrane Database Syst. Rev. 2, CD009121; doi:10.1002/14651858.CD009121. pub2 